\title{
An improved proximity force approximation for electrostatics
}

\author{
César D. Fosco ${ }^{1,2}$, Fernando C. Lombardo ${ }^{3}$, and Francisco D. Mazzitelli ${ }^{1,3}$ \\ 1 Centro Atómico Bariloche, Comisión Nacional de \\ Energía Atómica, R8402AGP Bariloche, Argentina \\ 2 Instituto Balseiro, Universidad Nacional de Cuyo, \\ R8402AGP Bariloche, Argentina and \\ 3 Departamento de Física Juan José Giambiagi, FCEyN UBA, \\ Facultad de Ciencias Exactas y Naturales, Ciudad Universitaria, \\ Pabellón I, 1428 Buenos Aires, Argentina - IFIBA \\ (Dated: today)
}

\begin{abstract}
A quite straightforward approximation for the electrostatic interaction between two perfectly conducting surfaces suggests itself when the distance between them is much smaller than the characteristic lengths associated to their shapes. Indeed, in the so called "proximity force approximation" the electrostatic force is evaluated by first dividing each surface into a set of small flat patches, and then adding up the forces due two opposite pairs, the contribution of which are approximated as due to pairs of parallel planes. This approximation has been widely and successfully applied to different contexts, ranging from nuclear physics to Casimir effect calculations. We present here an improvement on this approximation, based on a derivative expansion for the electrostatic energy contained between the surfaces. The results obtained could be useful to discuss the geometric dependence of the electrostatic force, and also as a convenient benchmark for numerical analyses of the tip-sample electrostatic interaction in atomic force microscopes.
\end{abstract}

Corresponding author: F.D. Mazzitelli. EMAIL: fdmazzi@cab.cnea.gov.ar; TEL: 542944445151 Ext39; FAX 542944445299 


\section{INTRODUCTION}

A standard problem in electromagnetism is to compute the electrostatic force between conducting bodies, or its close relative: the calculation of the capacity of a system of conductors. The simplest example is the case of parallel plates separated by a distance much smaller than the characteristic size of the plates, which are held at a fixed electrostatic potential difference. Albeit not as straightforwardly as in that example, some other systems admit analytical exact solutions also; indeed, that is the case for two eccentric cylinders, for a cylinder in front of a plane, and also for a sphere in front of a plane.

This problem is also of considerable practical relevance in Electrostatic Force Microscopy (EFM) and its variants [1, which are based on the interaction between biased atomic force microscopy (AFM) tips and a sample. The same applies to the experimental determination of Casimir or gravitational forces between conducting bodies, as residual charges or potentials produce unwanted forces that must be subtracted in order to determine the sought-after force $[2]$.

Of course the electrostatic force between bodies of arbitrary shape can, in principle, be computed by solving numerically the Laplace equation with adequate boundary conditions. However, analytic or semi-analytic methods are always welcome, as ways to improve the understanding of the geometric dependence of the force, and also to be used as simple benchmarks of fully numerical computations. For instance, in the context of EFM/AFM, analytical models for the tips have been developed, and additional exactly solvable models have been found, like the case of an hyperboloid in front of a plane [3]. Generalized imagecharge methods have also been proposed [4], in which the tip and the sample are replaced by a set of fictitious charges, whose intensity and positions are found numerically.

An interesting analytical approach was introduced by B. Derjaguin in 1934, who developed an approximate method to compute the Van der Waals force between macroscopic bodies assumed to be close to each other [5, 6]. The approximation assumes that the surfaces of the bodies can be replaced by a set of flat patches and that at short distances the dominant contributions correspond to pairs of patches (one on each surface) which are closest to each other. Moreover, the interaction is supposed to be additive. In this way, it is possible

to compute the force between gently curved surfaces from the knowledge of the interaction energy for flat surfaces, as long as the radii of curvature of the surfaces are much larger than 
the minimum distance between bodies and when the surface normals in opposite patches are almost parallel. Later on, the same idea was applied to nuclear physics, under the name of Proximity Force Approximation (PFA) or Proximity Force Theorem, in order to compute the interaction between nuclei [7, 8]. It has also been widely used to compute Casimir (or retarded Van der Waals) forces between neutral macroscopic objects [2]. It should be clear that, mutatis mutandis, the Derjaguin approximation and its ulterior developments can also be applied to the analysis of electrostatic forces, at least between gently curved conducting surfaces.

In spite of the simplicity and usefulness of the PFA, for many years there was a stumbling block to its progress, since methods to asses its reliability, or to compute the next to leading order (NTLO) correction were lacking. This situation left, as the only alternative of assessing the PFA reliability, its comparison with the rather few examples for which an exact result was available.

In an attempt to improve that situation, we have recently shown [9], in the context of Casimir physics, that the PFA can be considered as the first term, in an expansion in derivatives of the surfaces shapes, of the interaction energy. In this way, it is now possible to improve the PFA by computing the NTLO corrections.

In this paper we carry out this idea in the context of electrostatics, as already suggested in [9]. Our aim is twofold: on the one hand, to show the potential usefulness of the improved PFA in computing electrostatic forces. On the other hand, we believe that this attempt to present the improved PFA in a simpler context, may help one to gain intuition about its applicability in more complex scenarios.

\section{PROXIMITY FORCE APPROXIMATION}

Let us assume, for simplicity's sake, that the system consists of a gently varying surface in front of a plane (both perfect conductors). The plane is at $z=0$, and we assume it to be grounded. The curved surface is described by a single function $z=\psi(x, y)$, and is assumed to be at a constant electrostatic potential $V$. We shall use the notation $x_{\|}=(x, y)$. The electrostatic energy contained between surfaces is then given by:

$$
U=\frac{\epsilon_{0}}{2} \int d^{2} x_{\|} \int_{0}^{\psi\left(x_{\|}\right)} d z|\mathbf{E}|^{2} .
$$


The force between the conductors can be obtained by computing the variation of $U$ under a rigid displacement of one of the surfaces, while the capacity of the system is given by $C=2 U / V^{2}$.

In the PFA, this system is replaced by a set of parallel plates (see Fig.1). The intensity of the electric field between parallel plates separated by a distance $\psi$ has the $z$-independent value $V / \psi$. Therefore, the electrostatic energy is approximated by

$$
U_{\mathrm{PFA}}=\frac{\epsilon_{0} V^{2}}{2} \int d^{2} x_{\|} \frac{1}{\psi} .
$$

When computing Van der Waals, nuclear or Casimir forces, the rather involved nature of the interaction considered can render the interpretation of the approximations involved in the PFA somewhat obscure. Here, on the contrary, the physical assumption is quite clear: the electric field between opposite patches is regarded as constant and directed along the line that joins both patches.

Eq.22 can be used to estimate the electrostatic energy in many interesting situations. To illustrate this point, let us consider a cylinder of length $L$ and radius $R$ in front of a plane, and let us denote by $a$ the minimum distance between surfaces. The function $\psi$, that describes the part of the cylindrical surface which is closer to the plane, reads:

$$
\psi(x)=a+R\left(1-\sqrt{1-\frac{x^{2}}{R^{2}}}\right),
$$

with $-x_{M}<x<x_{M}<R$ in order to cover the part of the cylinder which is closer to the plane. It is reasonable to assume that $x_{M} / R=O(1)<1$, and under this assumption the final result will not depend on $x_{M}$, as long as $R \gg a$, where the PFA is expected to give an accurate value of the electrostatic energy. Inserting Eq.(3) into Eq.(2), the integral can be computed in terms of elementary functions. Expanding that result for $a \ll R$ we obtain

$$
U_{\mathrm{PFA}}^{\mathrm{cp}} \approx \frac{\epsilon_{0} V^{2} L \pi}{\sqrt{2}} \sqrt{\frac{R}{a}}
$$

which is independent of $x_{M}$. Note that, when the cylinder is close to the plane, the electrostatic force is proportional to $a^{-3 / 2}$.

For this geometry, the exact electrostatic interaction energy is known [10]:

$$
U^{\mathrm{cp}}=\frac{\pi L \epsilon_{0} V^{2}}{\operatorname{arccosh}\left(1+\frac{a}{R}\right)} .
$$




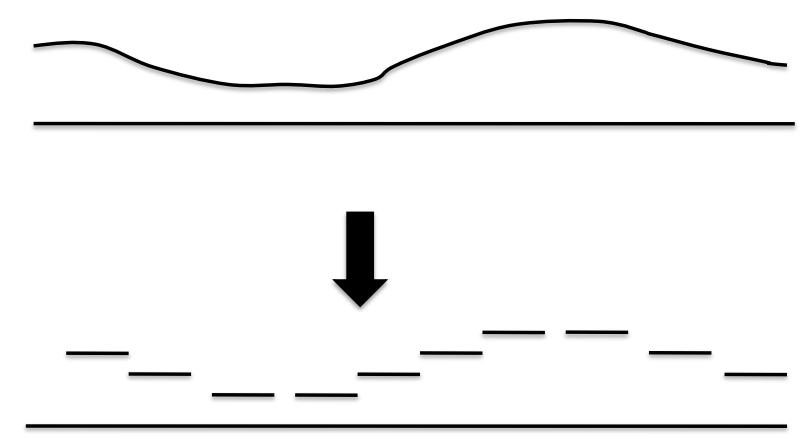

FIG. 1. In the Derjaguin approximation, the interaction between a gently curved surface and a plane is approximated by that of a set of parallel plates. For each pair of parallel plates, border effects are ignored.

In the limit of $a / R \ll 1$, this exact electrostatic energy indeed reduces to the PFA result given in Eq.(4). For later use, and to estimate the relevance of the corrections to the PFA, we expand the exact result including the NTLO in $a / R$ :

$$
U^{\mathrm{cp}} \approx \frac{\epsilon_{0} V^{2} L \pi}{\sqrt{2}} \sqrt{\frac{R}{a}}\left(1+\frac{1}{12} \frac{a}{R}\right) \equiv U_{\mathrm{NTLO}}^{\mathrm{cp}} .
$$

In Fig. 2 we plot the ratios $U^{\mathrm{cp}} / U_{\mathrm{PFA}}^{\mathrm{cp}}$ and $U^{\mathrm{cp}} / U_{\mathrm{NTLO}}^{\mathrm{cp}}$ as a function of $a / R$. We see that the NTLO produces a noticeable improvement of the PFA. We will now compute such improvement for an arbitrary geometry, of which the exact electrostatic energy in not necessarily known.

\section{IMPROVING THE PFA: DERIVATIVE EXPANSION}

In order to improve the PFA, we shall use the fact that the electrostatic energy may be thought of as a functional of the shape of the surface. This functional will be, in general, a non-local object, which becomes local when the surfaces are sufficiently close and parallel to each other. To interpolate between those two situations, we shall therefore assume that the electrostatic energy can be expanded in derivatives of $\psi$. Indeed, one can think of the condition $|\nabla \psi| \ll 1$, as the existence of a small, dimensionless parameter. This translates into the fact that the upper surface is almost parallel to the plane.

Including terms with up to two derivatives, the electrostatic energy has to be of the form:

$$
U_{\mathrm{DE}} \simeq \int d^{2} x_{\|}\left[V_{\mathrm{eff}}(\psi)+Z(\psi)|\nabla \psi|^{2}\right]
$$




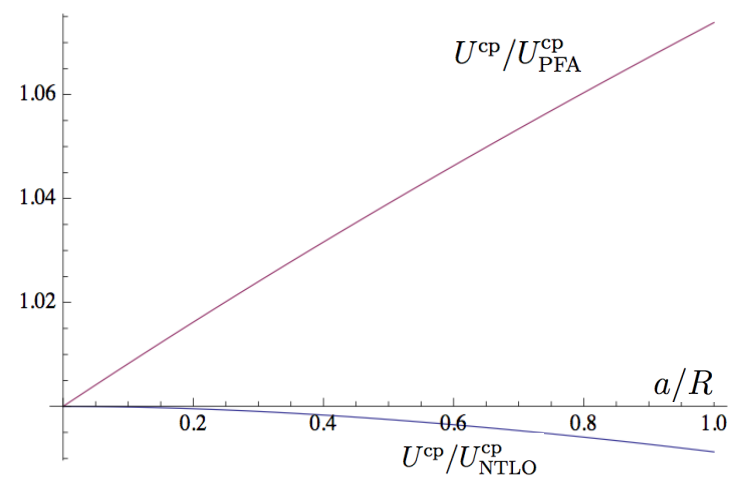

FIG. 2. Ratio of the exact energy and PFA (upper curve) for the cylinder in front of a plane, as a function of $a / R$. For the same geometry, the lower curve corresponds to the ratio of the exact energy and the approximation that includes the NTLO correction. The inclusion of the NTLO improves notably the approximation.

for some functions $V_{\text {eff }}$ and $Z$ [11]. The result must be proportional to $\epsilon_{0} V^{2}$, and must reproduce $U_{\mathrm{PFA}}$ for constant $\psi$. Moreover, as there are no other dimensional quantities beyond $\psi$, dimensional analysis imply that both functions $V_{\text {eff }}$ and $Z$ must be proportional to $\psi^{-1}$ :

$$
U_{\mathrm{DE}} \simeq \frac{\epsilon_{0} V^{2}}{2} \int d^{2} x_{\|} \frac{1}{\psi}\left(1+\beta_{\mathrm{em}}|\nabla \psi|^{2}\right)
$$

where $\beta_{\mathrm{em}}$ is the only (numerical) coefficient to be determined. Note that this coefficient is independent of the nature of the surface being considered; therefore, it may be obtained once and for all from its evaluation in a single case. Indeed, in order to obtain the coefficient $\beta_{\mathrm{em}}$, the simplest approach, when an exact solution of the problem is available, would be to read its value by expanding the exact solution. For instance, for the particular configuration of a cylinder in front of a plane, one can insert Eq.(3) into Eq.(8), perform the integrals, and expand the result in powers of $a / R$. Comparing this with the expansion of the exact result given in Eq.(6), it is straightforward to show that $\beta_{\mathrm{em}}=1 / 3$. Of course the same result could have been obtained from any other particular example.

Nevertheless, the coefficient $\beta_{\text {em }}$ can also be computed from first principles, as described in the next section. 


\section{SOLVING THE LAPLACE EQUATION}

It is perhaps more satisfying to prove that $\beta_{\mathrm{em}}=1 / 3$ without assuming a particular shape for the curved surface, but rather for a rather general class of surfaces. To that end it will be sufficient to derive it by assuming that $\psi\left(x_{\|}\right)=a+\eta\left(x_{\|}\right)$where $a$ is the mean value

of $\psi$, and $\left|\eta\left(x_{\|}\right)\right| \ll 1$. For this class of surfaces, the derivative expansion of the electrostatic energy reads

$$
U_{\mathrm{DE}} \simeq \frac{\epsilon_{0} V^{2}}{2 a} \int d^{2} x_{\|}\left[1+\left(\frac{\eta}{a}\right)^{2}+\beta_{\mathrm{em}}|\nabla \eta|^{2}+O\left(\eta^{3}\right)\right],
$$

and therefore he coefficient $\beta_{\mathrm{em}}$ can be read from the quadratic dependence of $U_{\mathrm{DE}}$ on the derivatives of $\eta$.

The electric field is given by $\mathbf{E}=-\nabla \phi$, where $\phi$ is the electrostatic potential, which satisfies the Laplace equation with the appropriate boundary conditions on the surfaces:

$$
\nabla^{2} \phi=0, \quad \phi_{z=0}=0, \quad \phi_{z=\psi}=V
$$

To proceed, we follow the approach of [12], to trade a simple boundary condition on a complex surface into a complex boundary condition on a flat surface. We expand the boundary condition on the upper surface as

$$
V=\phi\left(x_{\|}, a+\eta\right)=\phi\left(x_{\|}, a\right)+\eta \partial_{z} \phi\left(x_{\|}, a\right)+\frac{1}{2} \eta^{2} \partial_{z}^{2} \phi\left(x_{\|}, a\right)+\ldots,
$$

and we expand the electrostatic potential as

$$
\phi=\phi_{0}+\phi_{1}+\phi_{2}+\ldots
$$

where $\phi_{0}$ is the solution for parallel plates separated by a distance $a$, that is $\phi_{0}=V z / a$, and $\phi_{i}$ is assumed to be $O\left(\eta^{i}\right)$.

Inserting Eq.(12) into Eq.(11), we see that $\phi_{1}$ and $\phi_{2}$ must satisfy the following boundary conditions on $z=a$ :

$$
\begin{aligned}
\phi_{1}\left(x_{\|}, a\right) & =-\frac{V}{a} \eta\left(x_{\|}\right) \\
\phi_{2}\left(x_{\|}, a\right) & =-\eta\left(x_{\|}\right) \partial_{z} \phi_{1}\left(x_{\|}, a\right) .
\end{aligned}
$$

Thus, the initial problem has been converted into a problem of parallel plates for $\phi_{1}$ and $\phi_{2}$, with more involved boundary conditions. Up to quadratic order, the electrostatic energy reads

$$
U_{\mathrm{DE}} \simeq \frac{\epsilon_{0}}{2} \int d^{2} x_{\|} \int_{0}^{a+\eta} d z\left[\frac{V^{2}}{a^{2}}+\left|\nabla \phi_{1}\right|^{2}+2 \frac{V}{a} \partial_{z} \phi_{1}+2 \frac{V}{a} \partial_{z} \phi_{2}\right]
$$


It is easy to check that the last two terms in Eq.(14) do cancel:

$$
\int d^{2} x_{\|} \int_{0}^{a+\eta} d z\left(\partial_{z} \phi_{1}+\partial_{z} \phi_{2}\right)=\int d^{2} x_{\|} \eta\left(x_{\|}\right)=0,
$$

where we have used that $\left.\left(\phi_{1}+\phi_{2}\right)\right|_{a+\eta}=\eta+o\left(\eta^{2}\right)$ and that the mean value of $\eta$ vanishes.

Therefore the correction to the PFA is given solely by the term proportional to $\left|\nabla \phi_{1}\right|^{2}$. The order- $\eta$ solution $\phi_{1}$ is given by

$$
\phi_{1}\left(x_{\|}, z\right)=-\frac{V}{a} \int \frac{d^{2} k_{\|}}{(2 \pi)^{2}} e^{i k_{\|} x_{\|}} \tilde{\eta}\left(k_{\|}\right) \frac{\sinh k z}{\sinh k a} .
$$

Indeed, it is simple to check that it satisfies both the Laplace equation and the correct boundary condition (we are using the notation $k \equiv\left|k_{\|}\right|$). The solution for $\phi_{2}$ is similarlooking, although as already pointed out its explicit expression will not be necessary in order to evaluate the second-order energy.

Using the solution Eq. 16) we obtain:

$$
\int d^{3} x\left|\nabla \phi_{1}\right|^{2}=\frac{V^{2}}{a^{2}} \int \frac{d^{2} k_{\|}}{(2 \pi)^{2}}\left|\tilde{\eta}\left(k_{\|}\right)\right|^{2} F\left(k_{\|}\right),
$$

where

$$
F\left(k_{\|}\right)=\int_{0}^{a} d z \frac{k^{2}}{\sinh ^{2} k a}\left(1+2 \sinh ^{2} k z\right) \simeq \frac{1}{a}+\frac{a k^{2}}{3} .
$$

(note that the integration in $z$ runs up to $a$ instead of $a+\eta$ since the integrand is already of order $\left.\eta^{2}\right)$. Therefore

$$
\frac{\epsilon_{0}}{2} \int d^{3} x\left|\nabla \phi_{1}\right|^{2} \simeq \frac{\epsilon_{0} V^{2}}{2 a} \int d^{2} x_{\|}\left[\left(\frac{\eta^{2}}{a^{2}}\right)+\frac{1}{3}|\nabla \eta|^{2}\right],
$$

which is of the form of Eq.(9), and shows that $\beta_{\mathrm{em}}=1 / 3$. The final result is then

$$
U_{\mathrm{DE}} \simeq \frac{\epsilon_{0} V^{2}}{2} \int d^{2} x_{\|} \frac{1}{\psi}\left[1+\frac{1}{3}|\nabla \psi|^{2}\right],
$$

as expected from the example of the cylinder in front of a plane. Eq. 20) is the main result of this paper. The vertical component of the force on the upper surface can be computed by taking the variation of the energy under an infinitesimal translation $\psi \rightarrow \psi+\delta$, that is

$$
F_{z} \simeq \frac{d U_{\mathrm{DE}}}{d \delta} \simeq-\frac{\epsilon_{0} V^{2}}{2} \int d^{2} x_{\|} \frac{1}{\psi^{2}}\left[1+\frac{1}{3}|\nabla \psi|^{2}\right] .
$$

Note that, as the conductors are not isolated (they are kept at a fixed potential difference), the force is given by the derivative of the energy with respect to the position, with a positive sign. 


\section{TIP-SAMPLE ELECTROSTATIC INTERACTION}

In this section we use the improved PFA to analyze the problem of the tip-sample electrostatic force in an AFM. Typical geometries to model the interaction are sphere-plane, paraboloid-plane, hyperboloid-plane, and sphere-ended cone in front of a plane, among others [3]. In all these cases, it is possible to obtain the electrostatic force using the improved PFA. The results will be accurate when the tip is sufficiently close to the sample.

\section{A. Sphere-plane}

As a first example, we describe the case of a sphere of radius $R$, in front of a plane at a minimum distance $a(a \ll R)$. The surface of the sphere cannot be described by a single valued function $z=\psi\left(x_{\|}\right)$. We will just consider the region of the sphere which is closer to the plane, and we will see that it still give quantitatively adequate results even beyond the lowest order. The function $\psi$ is

$$
\psi(\rho)=a+R\left(1-\sqrt{1-\frac{\rho^{2}}{R^{2}}}\right),
$$

where we are using polar coordinates $(\rho, \phi)$ in the plane $(x, y)$. This function describes the hemisphere when $0 \leq \rho \leq R$. The derivative expansion will be well defined if we restrict the integrations to the region $0 \leq \rho \leq \rho_{M}<R$. As in the example of the cylinder in front of a plane, described in Section II, we will assume that $\rho_{M} / R=O(1)<1$. Inserting this expression for $\psi$ into the derivative expansion for the electrostatic energy Eq. 20], one can perform explicitly the integrations and obtain an analytic expression $U_{\mathrm{DE}}\left(\rho_{M}, a, R\right)$. Expanding the result for $a / R \ll 1$ we obtain

$$
U_{\mathrm{DE}} \simeq U_{\mathrm{PFA}}^{\mathrm{sp}}+f_{1}+\frac{1}{3} \frac{a}{R}\left(U_{\mathrm{PFA}}^{\mathrm{sp}}+f_{2}\right)
$$

where the leading contribution is given by

$$
U_{\mathrm{PFA}}^{\mathrm{sp}}=-\pi V^{2} \epsilon_{0} R \log (2 a / R),
$$

and $f_{1,2}$ are functions of $\rho_{M} / R$. Being independent of $a, f_{1}$ can be discarded if one is interested in computing the force between the sphere and the plane. The term proportional to $f_{2}$ gives a next to NTLO contribution to the force. Therefore we write the final result as

$$
U_{\mathrm{DE}} \simeq U_{\mathrm{PFA}}^{\mathrm{sp}}\left(1+\frac{1}{3} \frac{a}{R}\right)
$$


Note that, as long as $a \ll R$, the force will not depend on $\rho_{M}$ :

$$
F_{z} \simeq \frac{d U_{\mathrm{DE}}}{d a}=-\frac{\pi V^{2} \epsilon_{0} R}{a}\left(1+\frac{1}{3} \frac{a}{R} \log \left(\frac{a}{R}\right)\right) .
$$

This result could also be obtained directly from Eq.21), avoiding the appearance of the $\rho_{M}$-dependent constant terms $f_{1,2}$.

As already mentioned, this geometric configuration admits an exact solution [14]; at short distances that exact result reduces to Eqs. 25) and (26), as discussed in Ref. [15] (we include some details in the Appendix).

Finally, it is worth to remark that at short distances the electrostatic force for this

configuration is proportional to $a^{-1}$, milder than the cylinder-plane geometry $\left(a^{-3 / 2}\right)$ and than the parallel plates $\left(a^{-2}\right)$. This is of course a consequence of the different effective areas of interaction in each case, and has its counterpart in Casimir physics [13]. It is a simple property that could be emphasized in elementary discussions of electrostatics.

\section{B. Paraboloid-plane}

For the case of a paraboloid-like tip, the surface is described by

$$
\psi(\rho)=a+\frac{\rho^{2}}{2 R},
$$

with $0 \leq \rho \leq \rho_{\mathrm{M}}<R . \kappa=R^{-1}$ is the local curvature at the bottom of the paraboloid. The derivative expansion for the electrostatic interaction energy is

$$
U_{\mathrm{DE}} \simeq U_{\mathrm{PFA}}^{\mathrm{sp}}\left(1-\frac{2}{3} \frac{a}{R}\right) .
$$

It is not surprising that the leading order coincides with that of the sphere-plane interaction, since both surfaces have the same curvature at the point closest to the plane. However, the NTLO corrections are different, and in fact have opposite signs. These corrections can therefore be used as benchmarks of numerical calculations, since the short distance regime is typically the complex from a computational point of view.

\section{Hyperboloid-plane}

In this case the surface is described by the function

$$
\psi(\rho)=a-R_{1}+R_{1} \sqrt{1+\frac{\rho^{2}}{R_{2}^{2}}},
$$


where the curvature $\kappa$ at the bottom of the hyperboloid is $\kappa=R^{-1}$, with $R=R_{2}^{2} / R_{1}$, and $R_{1} / R_{2}$ is its asymptotic slope.

As in the previous examples, the derivative expansion of the electrostatic energy can be computed in terms of elementary functions. The result is

$$
U_{\mathrm{DE}} \simeq U_{\mathrm{PFA}}^{\mathrm{sp}}\left[1-\left(\frac{2}{3}-\frac{R_{2}^{2}}{R_{1}^{2}}\right) \frac{a}{R}\right]
$$

It is remarkable that sign of the NTLO correction depends on the parameters of the hyperboloid, and vanishes for the particular asymptotic slope $R_{1} / R_{2}=\sqrt{3 / 2}$.

\section{GENERALIZATIONS}

The results presented so far can be generalized in several directions:

\section{A. Beyond the next to leading order}

It is in principle possible to extend the results in order to include more derivatives of the shape function. For example, coming back to the case of a surface in front of a plane, the improved PFA would be, including the next to NTLO corrections:

$$
\begin{aligned}
U_{\mathrm{DE}} & =\frac{\epsilon_{0} V^{2}}{2} \int d^{2} x_{\|} \frac{1}{\psi}\left(1+\frac{1}{3}|\nabla \psi|^{2}+\gamma_{1}|\nabla \psi|^{4}+\gamma_{2} \psi|\nabla \psi|^{2} \nabla^{2} \psi\right. \\
& \left.+\gamma_{3} \psi^{2} \nabla^{2} \psi \nabla^{2} \psi+\gamma_{4} \psi^{2} \partial_{\alpha} \partial_{\beta} \psi \partial_{\alpha} \partial_{\beta} \psi+\gamma_{5} \psi^{3} \nabla^{2} \nabla^{2} \psi\right),
\end{aligned}
$$

where $\gamma_{1}, \gamma_{2}, \ldots \gamma_{5}$, are numerical coefficients that can be determined extending the calculation of Section IV up to $O\left(\eta^{4}\right)[16]$.

The structure of the improved PFA is useful to analyze its range of validity: the NTLO corrections are small when $|\nabla \psi| \ll 1$ or, in other words, when the curved surface is almost parallel to the plane. Higher order corrections will be negligible when, in addition to this

condition, the scale of variation of the shape of the surface is much larger than the local distance between surfaces.

\section{B. Flat surface coated with a dielectric layer}

The improved PFA can be extended to the case in which the sample (flat surface) is a perfect conductor coated with a layer of width $d$ of a material characterized with a constant 
permittivity $\epsilon$, as considered in the context of EFM [17]. Dimensional analysis implies that the derivative expansion for the electrostatic energy will be of the form

$$
U_{\mathrm{DE}} \simeq \frac{\epsilon_{0} V^{2}}{2} \int d^{2} x_{\|} \frac{1}{\psi}\left[\alpha\left(\frac{\epsilon}{\epsilon_{0}}, \frac{d}{\psi}\right)+\beta\left(\frac{\epsilon}{\epsilon_{0}}, \frac{d}{\psi}\right)|\nabla \psi|^{2}\right]
$$

for some functions $\alpha$ and $\beta$. We do not dwell here with the determination of the function $\beta$, but just want to remark that $\alpha$ can be obtained by elementary considerations for a parallel-plate capacitor with a dielectric layer:

$$
\alpha\left(\frac{\epsilon}{\epsilon_{0}}, \frac{d}{\psi}\right)=\frac{1}{1-\frac{d}{\psi}\left(1-\frac{\epsilon_{0}}{\epsilon}\right)} .
$$

\section{Two gently curved surfaces}

For the case of the Casimir energy, the result of Ref. [9] has been generalized to the case of two gently curved surfaces in Ref.[18]. This generalization can also be applied to the electrostatic case. Let us assume that the surfaces are described by two functions $\psi_{1}\left(x_{\|}\right)$ and $\psi_{2}\left(x_{\|}\right)$that measure the height of the surfaces with respect to a reference plane $\Sigma$. The derivative expansion for the electrostatic energy reads, in this case:

$$
\begin{aligned}
U_{\mathrm{DE}}\left[\psi_{1}, \psi_{2}\right] & =\frac{\epsilon_{0} V^{2}}{2} \int_{\Sigma} d^{2} x_{\|} \frac{1}{\psi}\left[1+\beta_{1}\left|\nabla \psi_{1}\right|^{2}\right. \\
& \left.\left.+\beta_{2}\left|\nabla \psi_{2}\right|^{2}+\beta_{\times} \nabla \psi_{1} \cdot \nabla \psi_{2}+\beta_{-} \hat{\mathbf{z}} \cdot \nabla \psi_{1} \times \nabla \psi_{2}\right)+\cdots\right],
\end{aligned}
$$

where $\psi=\left|\psi_{2}-\psi_{1}\right|$ is the height difference, and dots denote higher derivative terms.

As the energy must be invariant under the interchange of $\psi_{1}$ and $\psi_{2}, \beta_{1}=\beta_{2}$ and $\beta_{-}=0$. Moreover, in order to reproduce the previous result we must have $\beta_{1}=\beta_{2}=1 / 3$. Finally, the coefficient $\beta_{\times}$can be determined using the fact that the energy should be invariant under a tilt of the reference surface $\Sigma$ [18] or, alternatively, under a simultaneous rotation of both surfaces. For an infinitesimal rotation of each surface with an angle $\epsilon$ in the plane $(x, z)$ we have $\delta \psi_{i}=\epsilon\left(x+\psi_{i} \partial_{x} \psi_{i}\right)$, for $i=1,2$. In order to simplify the calculation we can assume that, initially, $\psi_{1}=0$ and that $\psi_{2}$ is only a function of $x$. Computing explicity the variation of $U_{D E}$ to linear order in $\epsilon$ one can show that

$$
\delta U_{\mathrm{DE}}=0 \Rightarrow \beta_{\times}=\frac{1}{3},
$$

and therefore

$$
U_{\mathrm{DE}}\left[\psi_{1}, \psi_{2}\right]=\frac{\epsilon_{0} V^{2}}{2} \int_{\Sigma} d^{2} x_{\|} \frac{1}{\psi}\left[1+\frac{1}{3}\left(\left|\nabla \psi_{1}\right|^{2}+\left|\nabla \psi_{2}\right|^{2}+\nabla \psi_{1} \cdot \nabla \psi_{2}\right)\right] .
$$


While the equality $\beta_{1}=\beta_{2}$ is valid for any interaction (as long as the surfaces are identical), the fact that $\beta_{\times}=\beta_{1}$ is valid only for the particular case of the electrostatic interaction, in which the leading term is proportional to $\psi^{-1}$ (i.e. is not valid for the Casimir energy, that is proportional to $\psi^{-3}$ for parallel plates).

Computing the variation of the electrostatic energy Eq. (34) with respect to translations or rotations of one of the surfaces, it is possible to obtain the vertical and lateral components of the force, as well as the torque produced by the presence of the other surface.

\section{FINAL REMARKS}

The PFA is a very useful approximation introduced by Derjaguin many years ago to compute Van der Waals forces between macroscopic bodies. The approximation has been subsequently widely used in rather different contexts, like nuclear physics and the Casimir

effect. Very recently, the approximation has been interpreted as the leading order in a derivative expansion of the energy, and this interpretation allowed to compute, for the first time, the NTLO corrections in a systematic way. In this paper we have pointed out that the improved PFA is a powerful tool which may also be used to compute the electrostatic interaction between conducting surfaces. Indeed, with this improved approximation, it is possible to compute the electrostatic force with high accuracy when the surfaces are sufficiently close to each other.

Moreover, given the simplicity of the expressions for the energy and the force, the NTLO could be used to test numerical methods aimed at a calculation of the force for arbitrary surfaces.

\section{NOTE ADDED:}

While this paper was under review, the revised version of Ref.[18] was published [19]. In the new version, the authors mentioned that they have successfully applied the gradient expansion to analyze the electrostatic interaction between a sphere and a plane, although there are no details of the calculations. 


\section{ACKNOWLEDGEMENTS}

This work was supported by ANPCyT, CONICET, UBA and UNCuyo.

\section{APPENDIX}

The electrostatic problem of a sphere of radius $R$ in front of a plane can be solved exactly: if the potential difference between the plane and the sphere is $V$, the electrostatic energy is given by 14

$$
U^{\mathrm{sp}}=2 \pi \epsilon_{0} R V^{2} \sinh \Gamma \sum_{n \geq 1} \frac{1}{\sinh (n \Gamma)},
$$

where $\cosh \Gamma=1+a / R$. In order to obtain an analytic expression in the limit $\Gamma \rightarrow 0$ we write

$$
\begin{aligned}
S \equiv \sum_{n \geq 1} \frac{1}{\sinh (n \Gamma)} & =S-\int_{1}^{\infty} \frac{d n}{\sinh (n \Gamma)}+\frac{1}{\Gamma} \ln (\operatorname{coth} \Gamma) \\
& =\frac{\gamma}{\Gamma}+\frac{1}{\Gamma} \ln (\operatorname{coth} \Gamma)+O(\Gamma),
\end{aligned}
$$

where $\gamma=0.5772$. Replacing this expression into Eq.(37) and expanding the result for small $a / R$ we obtain

$$
U^{\mathrm{sp}} \simeq-\pi \epsilon_{0} a V^{2} \ln (2 a / R)\left\{1+\frac{1}{3} \frac{a}{R}+O\left(\frac{a / R}{\ln (a / R)}\right)\right\},
$$

where we omited an irrelevant constant term. This result coincides, up to the NTLO, with the one obtained using the derivative expansion Eq.(25).

[1] C. C. Williams, W. P. Hough, and A. Rishton, Appl. Phys. Lett. 55, 203 (1989); A. Gil, J. Colchero, J. Gomez-Herrero, and A. M. Baro, Nanotechnology 14, 332 (2003); J. Hu, X.-D. Xiao, and M. Salmeron, Appl. Phys. Lett. 67, 476 (1995).

[2] M. Bordag, G.L. Klimchitskaya, U. Mohideen, and V. M. Mostepanenko, Advances in the Casimir Effect, Oxford University Press, Oxford, 2009.

[3] M. Lhernould, A. Delchambre, S. Regnier, P. Lambert, Applied Surface Science 253, 6203 (2007). 
[4] G. Mesa and J. J. Sáenz, Appl. Phys. Lett. 69, 1169 (1996); S. Gómez-Moivas, L. S. Froufe, R. Carminati, J. J. Greffet, and J. J. Sáenz, Nanotechnology 12, 496 (2001).

[5] B.V. Derjaguin, Koll. Z. 69, N2, 155 (1934); B. V. Derjaguin and I. I. Abrikosova, Sov. Phys. JETP 3, 819 (1957); B. V. Derjaguin, Sci. Am. 203, 47 (1960).

[6] J.N. Israelachvili, Intermolecular and Surface Forces, Academic Press Inc., San Diego (1998).

[7] J. Blocki, J. Randrup, W. J. Swiatecki, and C. F. Tsang, Ann. Phys. NY 105, 427 (1977); J. Blocki and W. J. Swiatecki, Ann. Phys. NY 132, 53 (1981).

[8] W. D. Myers and W. J. Swiatecki, Phys. Rev. C 62, 044610 (2000); I. Dutt and R. K. Puri, Phys. Rev. C 81, 064609 (2010).

[9] C. Fosco, F. Lombardo and F. Mazzitelli, Phys. Rev. D 84, 105031 (2011).

[10] L. D. Landau and E.M. Lifshitz, Electrodynamics of continuous media, Pergamon Press, Oxford (1984).

[11] We use the typical notation for the derivative expansion in Quantum Field Theory, in which $V_{\text {eff }}$ is the effective potential and $Z$ the wave function renormalization.

[12] L. H. Ford and A. Vilenkin, Phys. Rev. D 25, 2569 (1982).

[13] D. Dalvit, F. Lombardo, F. Mazzitelli, and R. Onofrio, Europhys. Lett. 67, 517 (2004).

[14] W. R. Smythe, Static and Dynamic Electricity, McGraw-Hill, New York (1968).

[15] F.D. Mazzitelli, F.C. Lombardo, and P. I. Villar, J. Phys.: Conf. Ser. 161, 012015 (2009).

[16] One could be tempted to obtain information about the coefficients $\gamma_{i}$ from the exact result of the cylinder in front of a plane. However, there is a subtle point here, because the next to NTLO corrections for this geometry depend on the quantity $x_{M}$ introduced in Section II.

[17] K. S. Date, A. V. Kulkarni, and C. V. Dharmadhikari, e-Journal of Surface Science and Nanotechnology 9, 206 (2011).

[18] G. Bimonte, T. Emig, R. L. Jaffe, M. Kardar, Casimir forces beyond the proximity approximation, arXiv:1110.1082.

[19] G. Bimonte, T. Emig, R. L. Jaffe, M. Kardar, Europhys. Lett. 97, 50001 (2012). 